\title{
The functional post-op rehabilitation results in the trimalleolar ankle fracture: A one-year follow-up of the case
}

\author{
Nazim Tolgahan Yildiz ${ }^{1}$, Hikmet Kocaman ${ }^{2}$, Gürsoy Coşkun ${ }^{1}$, Nilgün Bek ${ }^{3}$ \\ ${ }^{I}$ Faculty of Physical Therapy and Rehabilitation, Hacettepe University, Ankara, Turkey \\ ${ }^{2}$ Department of Physiotherapy and Rehabilitation, Faculty of Health Sciences, Karamanoğlu Mehmetbey University, Karaman, Turkey \\ ${ }^{3}$ Department of Physiotherapy and Rehabilitation, Faculty of Health Sciences, Lokman Hekim University, Ankara, Turkey
}

Received: 2021-06-01.

Accepted: 2021-09-05

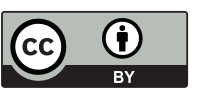

This work is licensed under a Creative Commons Attribution 4.0 International License

J Clin Med Kaz 2021; 18(5):88-91

Corresponding author:

Nazim Tolgahan Yildiz.

E-mail: tolgafty@gmail.com;

ORCID: 0000-0002-2404-2884.

\begin{abstract}
The aim of this study was to evaluate the one-year results of the effects of an eight-week functional rehabilitation program on pain, swelling, range of motion, balance, fear of movement, functional status of the ankle, and quality of life in a patient with a surgically treated trimalleolar ankle fracture. In the study, pain (Visual Analogue Scale), ankle joint swelling (Figure of eight methods), range of motion (Universal goniometer), static balance performance (Flamingo Balance Test), fear of movement (Tampa Kinesophobia Scale), functional status of the ankle (Foot Function Index) and quality of life (SF-36 Health Survey) were assessed. An eight-week functional rehabilitation program was applied to the patient after 8th week from surgery. Assessments were repeated before and after functional rehabilitation program, and at the one-year follow-up. At the end of the functional rehabilitation program and in oneyear follow-up, it was found that pain, swelling, and fear of movement decreased, range of motion, balance, functional status of the ankle, and quality of life increased. In trimalleolar ankle fractures, a well-planned functional physiotherapy and rehabilitation program after surgery can support surgical treatment by reducing the patient's symptoms and fear of movement, increasing functional capacity and quality of life.
\end{abstract}

Key words: ankle fracture, post-op treatment, physiotherapy, rehabilitation, one-year follow up

\section{Introduction}

Ankle fractures are among the most common fractures in the lower extremity [1]. These fractures are classified as unimalleolar, bimalleolar, or trimalleolar based on the number of malleolus involved in the fracture. Unimalleolar fracture refers to the fracture of either medial or lateral malleolus, while bimalleolar fracture describes fractures of both malleoli. The majority of ankle fractures comprise these fractures. A trimalleolar ankle fracture (TAF) is a type of complex ankle fracture that includes a posterior malleolar fracture in addition to the bimalleolar fracture. It is the rarest type that presents in $7 \%$ of all ankle fractures and generally occurs with highenergy trauma $[1,2]$.

While most of the ankle fractures are stable isolated malleolus fractures, some are unstable fractures. Unstable ankle fractures where malleoli are displaced are treated with surgical fixation with screws or plates [2]. In order to prevent instability and degenerative changes in posterior malleolus fractures that involve more than $25 \%$ of the articular surface (tibial plafond), fixation of the posterior fragment is provided by indirect method with lag screw or posterior direct approach [3]. After surgical treatment, patients often experience pain, swelling, stiffness, and loss of mobility [4]. It is crucial to apply an effective physiotherapy and rehabilitation program to prevent these problems and increase the functional status of the patient after surgery [2]. Reflex sympathetic dystrophy is a complex disorder affecting neural, vascular, bony, and soft tissue structures that can be seen in some patients with ankle fractures. It is characterized by pain, trophic changes (in structure in both deep and superficial tissues), sensory abnormalities, sweating and 
abnormal blood flow, stiffness, and functional impairment. A proper rehabilitation program can help reduce hypersensitivity and prevent contractures by providing cartilage nutrition and periarticular circulation in the joints [5].

The goals of the functional rehabilitation program (FRP) in ankle fracture are to treat the symptoms, restore or maintain range of motion (ROM), strength, proprioception, prevent the deterioration of the balance of strength and provide early functional mobility. Therapeutic modalities and exercise are components of ankle rehabilitation [6]. A very few studies have examined the effects of rehabilitation programs after surgically treated ankle fractures, and the reported results are controversial [1]. Also, there is no specific protocol for surgically treated TAF rehabilitation in the literature. The aim of this study was to evaluate the one-year follow-up results of the effects of an eightweek FRP on pain, swelling, ROM, balance, fear of movement, functional status of the ankle, and quality of life in a patient with a surgically treated TAF.

\section{Case presentation}

A 24-year-old female patient experienced an eversion left ankle (non-dominant foot) sprain as a result of a fall from a height. TAF in the ankle was detected by computed tomography (CT) (Figure 1). Surgical treatment was performed with the Open Reduction Internal Fixation method. With a standard incision of approximately $15 \mathrm{~cm}$ just above the lateral malleolus, the lateral malleolus and the posterior malleolus (antero-posterior lag screw) were fixed with the three and one cannulated screws, respectively (Figure 2). The patient's ankle was immobilized in a cast for eight weeks. When she applied to our clinic after the 8th week from surgery, had complaints of severe ankle pain during rest and activity, swelling, difficulty in walking, and climbing up and downstairs.

Figure 1 -Left ankle CT

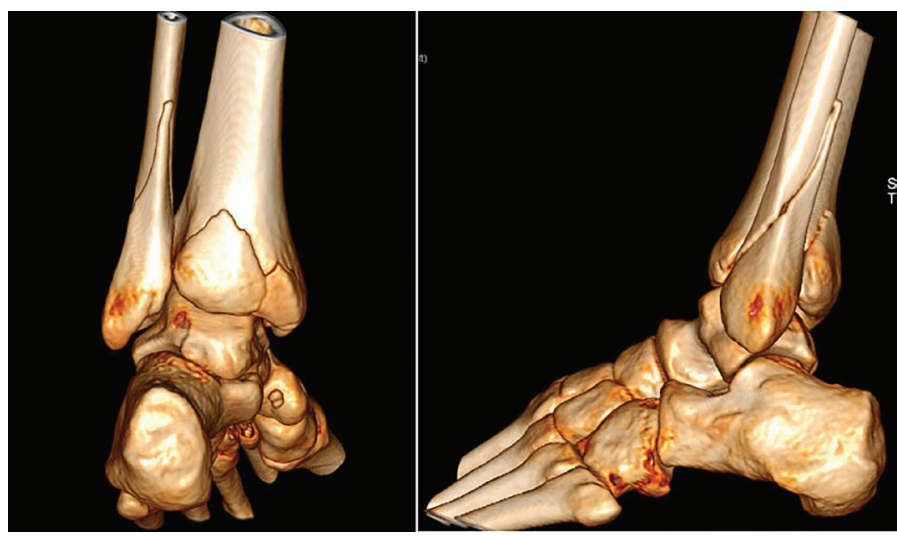

Figure 2 -Left ankle radiography
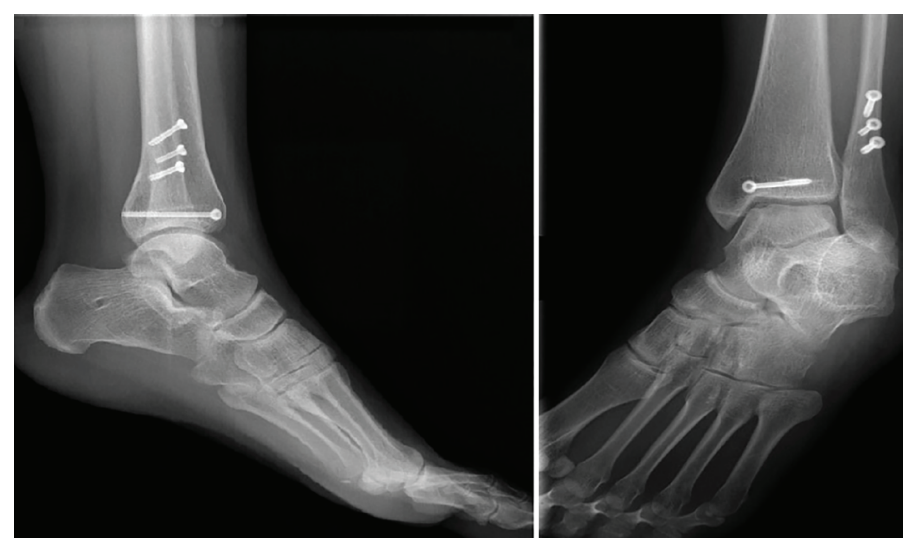

After obtaining verbal and written consent in the postop 8th week, the patient was included in the rehabilitation program after baseline assessments. The intensity of the pain at rest, activity and night of the peri ankle area was evaluated with the Visual Analogue Scale (VAS) [7]. The figure of eight shaped measurement method was used to evaluate the ankle joint swelling [8]. Dorsiflexion, plantar flexion, inversion and eversion passive range of motion of the ankle were measured with a universal goniometer. Static balance performance was evaluated for the left and right sides with the Flamingo Balance Test in eyes-open and eyes-closed positions. The score was recorded in seconds that it was able to maintain balance [9]. Fear of movement was assessed using the Tampa Kinesophobia Scale (TKS). TKS is a valid and reliable scale that consists of 17 questions used to assess fear of movement in problems related to the musculoskeletal system. The scale scores between 17 and 68 in total, and higher scores indicate that the fear of movement is high [10]. The Foot Function Index (FFI) was used to assess the functional status of the affected foot. The scale was developed to evaluate the impact of foot and ankle pathology on function in terms of pain, disability and activity restriction. The FFI is a self-administered scale consisting of 23 items divided into three subscales [11]. Quality of life was evaluated with the SF-36 Health Survey. SF-36 consists of two subsections as Physical Component Score (PCS) and Mental Component Score (MCS) and a total of 36 questions. High scores indicate a high quality of life [12]. Turkish validity and reliability of all scales used in this study were confirmed [10-12]. All assessments were repeated before and after the eight-week FRP and at one-year follow-up.

A total of 24 sessions of FRP, including eight weeks, three sessions a week, was applied to the patient. According to the patient's tolerance, the rehabilitation program was progressed through increasing activity speed and duration, varying the stability of the surface on which the patient, from double-leg to single-leg stance and from eyes-open to eyes-closed without any symptoms. In the first two weeks, the Transcutaneous Electrical Nerve Stimulation and vacuum interferential therapy was applied for 20 and 15 minutes respectively to decrease pain and swelling. Ankle mobilization and friction massage was performed to remove the adhesions and increase mobility in the ankle. Since the proximal tibiofibular joint is closely related to the ankle joint and can affect ankle mobility, mobilization for the proximal tibiofibular joint was added to the treatment program to increase the mobility of the ankle and proximal tibiofibular joint. Stretching exercises with the knee extended and flexed were included in the program to increase the Achilles tendon flexibility. As soon as a painless ROM was achieved, eccentric and concentric resistance strengthening exercises with Thera-band ${ }^{\circledR}$ elastic band were initiated and continued (Figure 3/A). In order to increase the balance and proprioception in the patient who felt poor balance confidence while weight-bearing to the left side, weight-bearing and squat exercises on stable and then unstable floors was added in the advanced stages of the rehabilitation program. The progress in these exercises was made from double-leg to single-leg stance and from eyes-open to eyes-closed (Figure 3/B).

It was found that there was a decrease in pain and ankle joint swelling, an increase in ROM and balance in the left ankle after treatment and at one-year follow-up (Table 1).

After treatment and at one-year follow-up, there was a decrease in the FFI sub-scale scores and the TKS score, and an increase in the scores of the PCS and MCS subsections of the SF-36 scale (Table 2). 
Figure 3 A -Resistance strengthening exercises with Theraband $\AA$, B: Balance and proprioceptive training on uneven surfaces

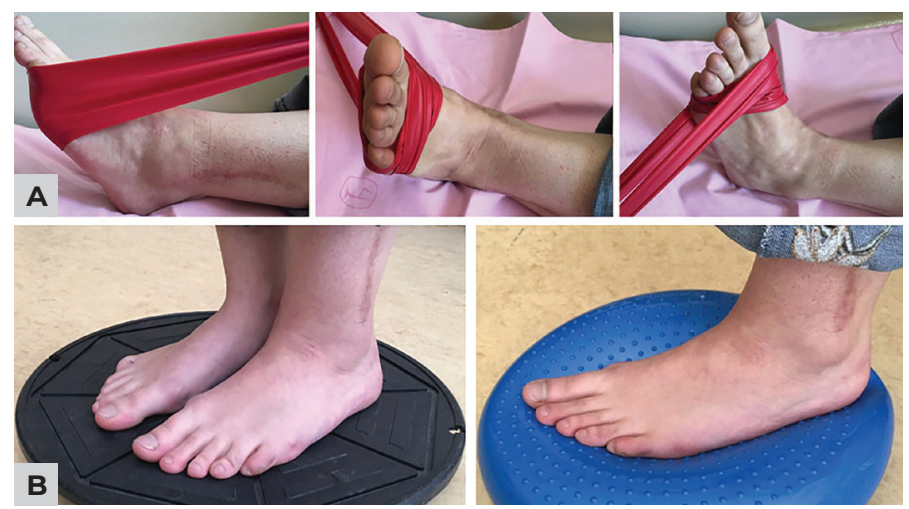

Table 1

Changes in pain, ankle joint swelling, ROM and balance parameters in the left ankle

\begin{tabular}{|l|l|l|l|l|l|}
\hline \multicolumn{2}{|c|}{} & \multirow{2}{*}{ Right } & \multicolumn{3}{|l|}{ Effected Foot } \\
\cline { 3 - 6 } \multicolumn{2}{|c|}{} & & BT & AT & 1 -YF \\
\hline \multirow{5}{*}{ VAS } & Pain at rest & 0 & 6.3 & 0 & 0 \\
\cline { 2 - 6 } & $\begin{array}{l}\text { Pain during } \\
\text { activity }\end{array}$ & 0 & 8.5 & 2.3 & 0 \\
\cline { 2 - 6 } & Pain at night & 0 & 8.2 & 0 & 0 \\
\hline Ankle joint swelling (cm) & 53 & 57.8 & 54.3 & 53.4 \\
\hline \multirow{3}{*}{ ROM $\left(^{\circ}\right)$} & Dorsiflexion & 16 & 5 & 13 & 15 \\
\cline { 2 - 6 } & Plantar flexion & 44 & 14 & 39 & 42 \\
\cline { 2 - 6 } & Eversion & 18 & 3 & 14 & 16 \\
\cline { 2 - 6 } & Inversion & 48 & 11 & 44 & 46 \\
\hline \multirow{3}{*}{$\begin{array}{l}\text { Balance } \\
\text { (second) }\end{array}$} & Eyes-open & 94.8 & 10.7 & 88.2 & 92.3 \\
\cline { 2 - 6 } & Eyes-closed & 55.6 & 5.5 & 49.4 & 52.7 \\
\hline
\end{tabular}

AT: After treatment, BT: Before treatment, ROM: Range of motion, VAS: Visual analog scale, 1-YF: One-year follow-up

Table 2

\begin{tabular}{|l|l|l|l|l|}
\hline \multicolumn{2}{|l|}{} & BT & AT & 1-YF \\
\hline \multirow{3}{*}{ FFI } & Pain (\%) & 58.7 & 17.5 & 0 \\
\cline { 2 - 6 } & Disability (\%) & 67.9 & 14.8 & 3.7 \\
\cline { 2 - 6 } & Activity restriction (\%) & 80 & 4.4 & 0 \\
\hline \multirow{2}{*}{ TKS } & PCS & 60 & 21 & 17 \\
\hline SF-36 Health Survey & 24.5 & 93.7 & 100 \\
\hline SF-36 Health Survey & MCS & 31.25 & 90 & 100 \\
\hline
\end{tabular}

AT: After treatment, BT: Before treatment, FFI: Foot Function Index, MCS: Mental Component Score, PCS: Physical Component Score, TKS: Tampa Kinesophobia Scale, 1-YF: One-year follow-up

\section{Discussion}

Foot and ankle pathologies lead to restriction of daily living activities, balance and walking problems. As a result of these problems, the functional status and quality of life of the person decrease [11]. Ankle fractures are the most common type of lower extremity fracture. TAF is the rarest type of ankle fracture. In TAF, it is considered essential to provide anatomic reduction with the appropriate surgical methods and to apply for an effective rehabilitation program after surgery in order to prevent instability and osteoarthritis in the future [2,3]. In the present study, an eight-week FRP was applied to the patient in which a TAF was surgically treated. It was observed that the pain and swelling in the ankle of the patient were decreased after the FRP, and completely disappeared in a one-year follow-up. It was found that ROM and balance increased after FRP, and these parameters reached approximately the same values as the right side at one-year follow-up. In addition, it was found that fear of movement decreased, functional status of the foot and quality of life increased.

Nilsson et al. [1] compared the 12-weeks individual training program with the usual care in patients with surgically treated ankle fractures. In this study, in which symptoms, functional status, ankle mobility, quality of life, and walking performance were evaluated, it was reported that the individual training program was superior to the usual care in patients under the age of 40. In addition, it was reported that more studies are needed to investigate the effectiveness of rehabilitation programs in patients with ankle fractures surgically treated [1]. Hong et al. [4] have been evaluated 31 patients with operatively managed TAF at 1 year postoperatively and found that $52.4 \%$ of patients had residual pain, $61.9 \%$ of patients had persistent ankle stiffness, $47.6 \%$ of patients had swelling. Also, they reported that $66.7 \%$ of patients were not able to return to sports, and $25 \%$ of patients were unable to do sports at all. In our study, at the one-year follow-up, it was seen that the patient had no residual pain and swelling. Also, the ROM of the left ankle increased and reached similar values with the right side. In the present study, the improvements we achieved in the symptoms, functional status, and quality of life of the patient indicate the importance of FRP in surgically treated TAF.

In the literature, it is reported that balance and proprioception training included in the rehabilitation program after ankle injury may have beneficial effects on postural stability, coordination, and proprioception [13]. For our case report, consistent with the literature, it was found that the patient's balance in eyesopen and eyes-closed positions increased after the FRP, and this improvement was preserved in a one-year follow-up. It has been reported that in patients with fractures, various complications, especially pain, caused by the fracture can cause fear of movement [14]. In the present report, considering the TKS score, it was found that the patient's fear of movement significantly decreased after the FRP and at the one-year followup. Following the surgically treated TAF, considering patients' fear of movement, and encouraging them to move, and weightbearing the affected side can positively affect the rehabilitation process.

In the present study, we found that after the eight-week FRP, the foot function improved according to FFI subscales, and this improvement was preserved in a one-year follow-up. Our reason to use the SF-36 to evaluate the quality of life in our study was that it has been widely used in orthopedics and had strong evidence of validity in evaluating the results of ankle fractures $[15,16]$. In our study, it was thought that the decrease in pain and fear of movement and the improvement of ROM, balance parameters, and foot function after FRP were effective in increasing the quality of life. The improvements in SF-36 scores in our study are consistent with previous reports $[15,16]$.

It can be considered as a limitation of this study that no measurement was made for proprioception assessment.

In trimalleolar ankle fracture, a well-planned functional physiotherapy and rehabilitation program after surgery can increase the success of surgical treatment by reducing the patient's symptoms and fear of movement, increasing functional capacity and quality of life.

Disclosures: There is no conflict of interest for all authors.

Acknowledgements: None.

Funding: None.

Patient informed consent: Obtained. 


\section{References}

1. Nilsson GM, Jonsson K, Ekdahl CS, Eneroth M. Effects of a training program after surgically treated ankle fracture: a prospective randomised controlled trial. BMC Musculoskelet Disord. 2009; 10(1):1-11. https://doi.org/10.1186/1471-2474-10-118

2. Michelson JD. Ankle fractures resulting from rotational injuries. J Am Acad Orthop Surg. 2003; 11(6):403-12. https://doi. org/10.5435/00124635-200311000-00004

3. Seyhan M. Fractures around the ankle [in Turkish]. Journal of the Turkish Society of Orthopaedics and Traumatology. 2013; 12(2):153-8. doi: 10.5606/totbid.dergisi.2013.17

4. Hong CC, Nashi N, Roy SP, Tan KJ. Impact of trimalleolar ankle fractures: how do patients fare post-operatively? Foot Ankle Surg. 2014; 20(1):48-51. https://doi.org/10.1016/j.fas.2013.10.001

5. Teasdall RD, Smith BP, Koman LA. Complex regional pain syndrome (reflex sympathetic dystrophy). Clin Sports Med. 2004; 23(1):145-55. doi:10.1016/S0278-5919(03)00090-5

6. Hsu RY, Bariteau J. Management of ankle fractures. R I Med J. 2013; 96(5):23-7.

7. Dones VC, Serra MAB, Kamus GOT, Esteban AC, Mercado AMS, Rivera RGA et al. The effectiveness of biomechanical taping technique on visual analogue scale, static maximum handgrip strength, and patient rated tennis elbow evaluation of patients with lateral epicondylalgia: A cross-over study. J Bodyw Mov Ther. 2019; 23(2):405-16. https://doi.org/10.1016/j.jbmt.2018.05.004

8. Mawdsley RH, Hoy DK, Erwin PM. Criterion-related validity of the figure-of-eight method of measuring ankle edema. J Orthop Sports Phys Ther. 2000; 30(3):149-53. https://www.jospt.org/doi/10.2519/jospt.2000.30.3.149

9. Hrysomallis C. Relationship between balance ability, training and sports injury risk. Sports Med. 2007; 37(6):547-56. https://doi. org/10.2165/00007256-200737060-00007

10. Tunca Yilmaz O, Yakut Y, Uygur F, Ulug N. Turkish version of the tampa scale for kinesiophobia and its test-retest reliability [in Turkish]. Turk J Physiother Rehabil. 2011; 22(1):44-9.

11. Kulunkoglu B, Firat N, Yildiz NT, Alkan A. Reliability and validity of the Turkish version of the foot function index in patients with foot disorders. Turk J Med Sci. 2018; 48(3):476-83. doi:10.3906/sag-1705-143

12. Kocyigit H, Aydemir O, Fisek G, Olmez N, Memis A. Reliability and validity of the Turkish version of form-36 (SF-36) [in Turkish]. Turkish journal of drugs \& therapeutics. 1999; 12(2):102-6.

13. Kovaleski JE, Kovaleski SJ, Pearsall AW. Functional rehabilitation after a lateral ankle injury. Int J Athl Ther Train. 2006; 11(3):52-5. https://doi.org/10.1123/att.11.3.52

14. Sengul YS, Unver B, Karatosun V, Gunal I. Assessment of pain-related fear in patients with the thrust plate prosthesis (TPP): due to hip fracture and hip osteoarthritis. Arch Gerontol Geriatr. 2011; 53(2):e249-52. https://doi.org/10.1016/j.archger.2011.05.007

15. Bhandari M, Sprague S, Hanson B, Busse JW, Dawe DE, Moro JK et al. Health-related quality of life following operative treatment of unstable ankle fractures: a prospective observational study. J Orthop Trauma. 2004; 18(6):338-45. https://doi.org/10.1097/00005131200407000-00002

16. Ponzer S, Nåsell H, Bergman B, Törnkvist H. Functional outcome and quality of life in patients with Type B ankle fractures: a two-year follow-up study. J Orthop Trauma. 1999; 13(5):363-8. https://doi.org/10.1097/00005131-199906000-00007 